\title{
CONFIABILIDADE E VALIDADE DE UM DINAMÔMETRO ISOMÉTRICO MODIFICADO NA AVALIAÇÃO DO DESEMPENHO MUSCULAR EM INDIVÍDUOS COM RECONSTRUÇÃO DO LIGAMENTO CRUZADO ANTERIOR
}

\author{
RELIABILITY AND VALIDITY OF A MODIFIED ISOMETRIC DYNAMOMETER IN \\ THE ASSESSMENT OF MUSCULAR PERFORMANCE IN INDIVIDUALS WITH \\ ANTERIOR CRUCIATE LIGAMENT RECONSTRUCTION
}

Rodrigo Antunes de Vasconcelos ${ }^{1}$, Débora Bevilaqua-Grossi², Antonio Carlos Shimano ${ }^{3}$, Cleber Jansen Paccola $^{4}$, Tânia Fátima Salvini ${ }^{5}$, Christiane Lanatovits Prado ${ }^{6}$, Wilson A. Mello Junior ${ }^{7}$

\section{RESUMO}

Objetivo: Analisar a confiabilidade e validade de um dinamômetro isométrico modificado (DIM) na avaliação dos déficits no desempenho muscular dos extensores e flexores do joelho em indivíduos normais e com reconstrução do LCA. Métodos: Foram convidados 60 voluntários do sexo masculino a participar do estudo, divididos em três grupos de 20 indivíduos: grupo controle (GC), grupo com reconstrução do LCA com tendão patelar (GTP) e grupo com reconstrução do LCA com tendões flexores (GTF). Todos os indivíduos realizaram teste isométrico dos extensores e flexores do joelho no DIM; os déficits de força muscular coletados foram comparados posteriormente com os testes realizados no Biodex System 3 operando no modo isométrico e isocinético nas velocidades de $60^{\circ} / \mathrm{s}$ e $180^{\circ} / \mathrm{s}$. Foram realizados cálculos de correlação intraclasse ICC para avaliar a confiabilidade do DIM, cálculos da especificidade, sensibilidade e coeficiente de concordância Kappa, respectivamente, para avaliar a validade do DIM em detectar déficits musculares e comparações intragrupos e intergrupos na realização dos quatro testes de força utilizando-se do método ANOVA. Resultados: O DIM demonstrou excelente confiabilidade teste-reteste e validade na

\section{ABSTRACT}

Objectives: The aim of this study was to evaluate the reliability and validity of a modified isometric dynamometer (MID) in performance deficits of the knee extensor and flexor muscles in normal individuals and in those with ACL reconstructions. Methods: Sixty male subjects were invited to participate of the study, being divided into three groups with 20 subjects each: control group $(G C)$, group of individuals with ACL reconstruction with patellar tendon graft (GTP, and group of individuals with ACL reconstruction with hamstrings graft (GTF). All individuals performed isometric tests in the MID, muscular strength deficits collected were subsequently compared to the tests performed on the Biodex System 3 operating in the isometric and isokinetic mode at speeds of $60 \%$ and $180 \mathrm{o} / \mathrm{s}$. Intraclass ICC correlation calculations were done in order to assess MID reliability, specificity, sensitivity and Kappa's consistency coefficient calculations, respectively, for assessing the MID's validity in detecting muscular deficits and intra- and intergroup comparisons when performing the four strength tests using the ANOVA method. Results: The modified isometric dynamometer (MID) showed excellent reliability and good validity in the as-

1 - Doutorando em Ciências Médicas, Área de Concentração Ortopedia, Traumatologia e Reabilitação pela Faculdade de Medicina de Ribeirão Preto - Universidade de São Paulo (FMRP-USP), São Paulo, Brasil.

2 - Professor Livre-Docente do Departamento de Biomecânica, Medicina e Reabilitação do Aparelho Locomotor da Faculdade de Medicina de Ribeirão Preto - Universidade de São Paulo (FMRP-USP), São Paulo, Brasil.

3 - Professor Doutor do Departamento de Biomecânica, Medicina e Reabilitação do Aparelho Locomotor da Faculdade de Medicina de Ribeirão Preto - Universidade de São Paulo (FMRP-USP), São Paulo, Brasil.

4 - Professor Titular do Departamento de Biomecânica, Medicina e Reabilitação do Aparelho Locomotor da Faculdade de Medicina de Ribeirão Preto - Universidade de São Paulo (FMRP-USP), São Paulo, Brasil.

5 - Professora Titular do Departamento de Fisioterapia da Universidade Federal de São Carlos (UFScar ), São Paulo, Brasil.

6 - Mestranda em Fisioterapia pelo Departamento de Fisioterapia da Universidade Federal de São Carlos (UFScar), São Paulo, Brasil

7 - Preceptor do programa de Residência em Ortopedia - Cirurgia do Joelho - Hospital Municipal Celso Pierro - Pontifícia Universidade Católica de Campinas (HMCPPUCC).

Trabalho realizado no Departamento de Biomecânica, Medicina e Reabilitação do Aparelho Locomotor da Faculdade de Medicina de Ribeirão Preto - Universidade de São Paulo (FMRP-USP). Correspondência: Rua Dom Bosco, 187, Bairro Taquaral - Campinas, SP. CEP:13076-060. Tels.: (19) 3251-0555 e (16) 3602-4413. E-mail: rodvasconcelos@hotmail.com 
avaliação do desempenho muscular dos extensores e flexores do joelho. Na comparação intergrupos. o GTP demonstrou déficits significativamente maiores dos extensores comparados com os grupos GC e GTF. Conclusão: Dinamômetros isométricos conectados em equipamentos de mecanoterapia podem ser uma alternativa para coletar dados referentes a déficits no desempenho muscular dos extensores e flexores do joelho em indivíduos com reconstrução do LCA.

Descritores - Joelho; Ligamento cruzado anterior; Protocolos; Estudos retrospectivos

\section{INTRODUÇÃO}

Os pesquisadores que estudam a articulação do joelho utilizam-se das unidades de medida torque, trabalho e potência para comparação entre grupos de indivíduos ou diferentes intervenções. Os estudos que analisam a influência da utilização de diferentes tipos de enxerto ${ }^{(1)}$, técni-

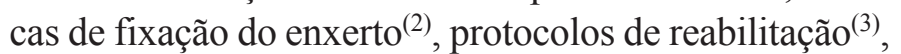
diferenças entre sexo ${ }^{(4)}$ no desempenho muscular e funcional em indivíduos submetidos a intervenção cirúrgica fornecem dados confiáveis que subsidiam a elaboração de protocolos de reabilitação por clínicos da área.

Os especialistas da área têm muito interesse na avaliação do desempenho dos músculos extensores e flexores do joelho porque a literatura fornece valores de referência para progressão das fases de reabilitação no pós-operatório de cirurgias de reconstrução do LCA, possibilitando o inicio da corrida, dos treinos de agilidade e retorno ao esporte competitivo baseado nos déficits encontrados no joelho lesado comparados ao joelho contralateral $^{(5)}$.

A avaliação de déficits musculares em atletas em fase de pré-temporada favorece intervenções preventivas através da correção dos déficits dos extensores e flexores do joelho ou o restabelecimento do equilíbrio agonista-antagonista $^{(6)}$. Entre os testes e equipamentos desenvolvidos para avaliação do desempenho muscular nos extensores e flexores do joelho, os dinamômetros isocinéticos computadorizados são considerados padrão ouro ${ }^{(7)}$.

Porém, dinamômetros isocinéticos são pouco acessíveis à maioria dos fisioterapeutas no Brasil devido ao alto custo do equipamento, não há fabricantes nacionais, e necessitam de espaço considerável de instalação.

Esses fatores tornam difícil o acesso por clínicos e pesquisadores de laboratórios ao desempenho muscular dos extensores e flexores do joelho em patologias de qualquer natureza. Uma alternativa a esse problema seria a utilização de testes isométricos que poderiam re- sessment of the performance of the knee extensor and flexor muscles groups. In the comparison between groups, the GTP showed significantly greater deficits as compared to the GTF and GC groups. Conclusion: Isometric dynamometers connected to mechanotherapy equipments could be an alternative option to collect data concerning performance deficits of the extensor and flexor muscles groups of the knee in subjects with ACL reconstruction.

Keywords - Knee, Anterior cruciate ligament; Protocols; Retrospective studies

produzir os déficits encontrados em testes realizados em dinamômetros isocinéticos computadorizados. Vários autores utilizaram um modelo de dinamômetro isométrico portátil chamado Hand-Held dynamometry ${ }^{(8-10)}$.

Esse equipamento é um dinamômetro manual digital que pode ser utilizado para mensurar torque isométrico em varias articulações realizando testes de força manual. Ele tem demonstrado boa confiabilidade intraexaminador na avaliação dos rotadores do ombro em sujeitos normais ${ }^{(11)}$, boa correlação com dinamômetros isocinéticos na avaliação de força muscular dos rotadores em ombros de pacientes com sequelas neurológicas ${ }^{(12)}$ e boa concordância na avaliação dos extensores do joelho em paciente geriátricos ${ }^{(13)}$. No entanto, para avaliar a desempenho muscular dos membros inferiores de indivíduos fisicamente ativos, o dinamômetro manual digital apresenta muitas desvantagens, pois não há estabilização do paciente e necessita de examinador forte para manter a angulação da articulação a ser testada durante a realização do exame. Esses dois fatores levaram a relatos de pobre confiabilidade na avaliação de potentes grupos musculares como o quadríceps ${ }^{(14)}$ e pobre correlação tem sido observada entre o dinamômetro manual digital e dinamômetros isocinéticos computadorizados na avaliação de paciente com status pós-operatório do joelho ${ }^{(15)}$. Essas conclusões podem estar relacionadas com o tipo de metodologia utilizada para avaliar e coletar dados referentes ao desempenho muscular. No entanto, há a necessidade de desenvolver equipamentos que possam utilizar sistema de estabilização do paciente, ajustes de ângulo articular para coletar dados referentes a déficits musculares significativos, possibilitando intervenção direcionada e que pudessem ser facilmente reproduzidos em diferentes centros clínicos e de pesquisa. Para isso é necessário avaliar critérios de validade e confiabilidade entre déficits no desempenho muscular isométrico e isocinético coletados em diferentes tipos de dinamômetros. 
Os objetivos do presente estudo foram avaliar a confiabilidade intraexaminador do equipamento de dinamometria isométrica modificada (DIM) e sua validade para classificar déficits no desempenho muscular dos grupos extensores e flexores do joelho em indivíduos normais e indivíduos submetidos à reconstrução do LCA tendo como exame referência o dinamômetro Biodex System 3.

\section{MÉTODOS}

Três grupos envolvendo um total de 60 voluntários do sexo masculino com idade entre 18 e 40 anos foram recrutados via convite verbal através de contato telefônico. O primeiro, denominado de grupo controle (GC), consistiu de 20 voluntários sem história prévia de lesões no joelho e que participavam de atividades esportivas amadoras. O segundo, denominado grupo tendão patelar (GTP), foi formado por 20 voluntários submetidos à reconstrução do LCA com enxerto autólogo do tendão patelar. O terceiro grupo foi constituído por 20 voluntários submetidos à reconstrução do LCA com enxerto autólogo dos tendões flexores (semitendinosus e gracilis); este foi denominado grupo tendões flexores (GTF). Todos os voluntários dos grupos GTP e GTF foram submetidos à cirurgia pelos cirurgiões ortopedistas do Grupo de Cirurgia do Joelho do Hospital Municipal Celso Pierro - Pontifícia Universidade Católica de Campinas. As características gerais dos participantes dos três grupos e detalhes do status pós-operatório dos grupos GTP e GTF estão descritos na tabela 1.

Os critérios de inclusão do grupo (GC) foram: ausência de histórias prévias de lesões no joelho de qualquer natureza, ausência de desordens neuromusculares, diferenças de frouxidão ligamentar entre joelhos $<3 \mathrm{~mm}$ avaliados pelo $K T$ 1000, Os critérios de inclusão do grupo GTP e GTF foram: seis meses de pós-operatório completos, ausência de sinais inflamatórios, marcha normal, ausência de desordens neuromusculares e sem queixas de instabilidade em Avds. Os critérios de exclusão para todos os grupos foram: histórico de lesões ligamentares bilaterais, cirurgias de reconstrução ligamentar de qualquer natureza prévias no joelho, fraturas de qualquer natureza nos membros inferiores, rupturas ligamentares combinadas, osteoartrose avançada nas articulações femoropatelar ou tibiofemoral com evidente desvio de eixo articular

Todos os voluntários receberam instruções por escrito detalhadas de como seria o teste e assinaram termo de consentimento livre e esclarecido concordando em participar do estudo. O projeto foi aprovado pelo Co- mitê de Ética do Hospital das Clinicas da Faculdade de Medicina de Ribeirão Preto- Universidade de São Paulo, oficio n ${ }^{0} 2977 / 2007$.

A avaliação subjetiva de Lysholm $^{(16)}$ foi utilizada para caracterizarmos funcionalmente a amostra. Essa escala é amplamente utilizada e foi validada para a língua portuguesa $^{(17)}$. Para avaliação do nível esportivo dos voluntários nos três grupos foi utilizada a escala de Tegner ${ }^{(18)}$.

O artrômetro KT 1000 (Medmetric, San Diego, CA) foi utilizado para avaliar todos os grupos; no grupo $\mathrm{GC}$ o equipamento determinou o critério de inclusão e exclusão dos participantes no estudo. Nos grupos GTP e GTF, o KT 1000 foi utilizado para determinar a estabilidade clinica do joelho operado.

Inicialmente, todos os participantes foram submetidos a uma avaliação clinica. Na avaliação clinica, além da coleta dos dados antropométricos, o voluntário foi submetido a algumas partes do exame físico, que compreende verificação da amplitude do movimento passiva (ADM) perimetria de coxas, e preenchimento do questionário de Lysholm e Tegner.

Após a avaliação clínica, os voluntários foram submetidos ao exame de artrometria KT 1000, através do teste máximo manual (TMM). Foram realizados três TMM e o maior valor foi anotado na ficha de avaliação clinica. No grupo GTP e GTF, o joelho não lesado sempre foi testado primeiro e, no grupo controle, o joelho não dominante.

Após a realização do TMM, todos os voluntários realizaram um aquecimento de cinco minutos em uma bicicleta ergométrica, seguido de três séries de 30 segundos de alongamentos tradicionais para quadríceps e isquiotibiais.

Tabela 1 - Características gerais dos voluntários distribuídos nos três grupos analisados GC, GTP e GTF

\begin{tabular}{|c|c|c|c|}
\hline Variáveis & Grupo GC & Grupo GTP & Grupo GTF \\
\hline Amostra (N) & 20 & 20 & 20 \\
\hline Idade (anos) & $24,95( \pm 5,18)$ & $32,6( \pm 7,76)$ & $27,55( \pm 6,88)$ \\
\hline Altura $(\mathrm{cm})$ & $175,1( \pm 7,35)$ & $176,2( \pm 8,48)$ & $179,5( \pm 8,99)$ \\
\hline Peso (kg) & $75,8( \pm 8,7)$ & $85,5( \pm 14,3)$ & $79,9( \pm 8,7)$ \\
\hline Perimetria * & $0,8( \pm 0,6)$ & $1,42( \pm 1,1)$ & $1,35( \pm 1,3)$ \\
\hline Escala de Tegner & 5,5 & 6,2 & 6,7 \\
\hline Tempo PO (meses) & 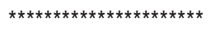 & $8,5( \pm 5,45)$ & $8,5( \pm 4,00)$ \\
\hline Reabilitação supervisionada & 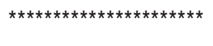 & $10 / 20$ & $15 / 20$ \\
\hline Reabilitação domiciliar & 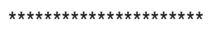 & $10 / 20$ & $5 / 20$ \\
\hline
\end{tabular}

Legendas: GC = grupo controle; GTP = grupo tendão patelar; GTF = grupo tendões flexores. * Perimetria demonstrando valores de diferenças entre coxas coletada a $15 \mathrm{~cm}$ do polo superior da patela

Fonte: FMRP-USP 
Para a coleta dos dados referentes ao desempenho funcional dos participantes, foi desenvolvido um equipamento de dinamometria isométrica modificada (DIM). Esse equipamento foi desenvolvido para coletar o torque extensor e flexor do joelho através de contrações isométricas.

Para tornar possível a quantificação dos déficits no desempenho muscular dos participantes envolvidos, foram utilizados dois dinamômetros analógicos de tração Kratos $^{\circledR}$ modelo LDC (Kratos Equipamentos Industriais Ltda.) (Figura 1a). Os dinamômetros de tração são constituídos de caixa de alumínio fundido, possuem capacidade de 1 a 100kgf, oferecendo $2 \%$ de precisão do ponto lido e são fornecidos com certificado de calibração rastreado pelo Registro Brasileiro de Calibrações (RBC). Os dinamômetros de tração foram instalados na parte posterior do equipamento de mecanoterapia, onde eram alocados os pesos originalmente (Figura 1b).

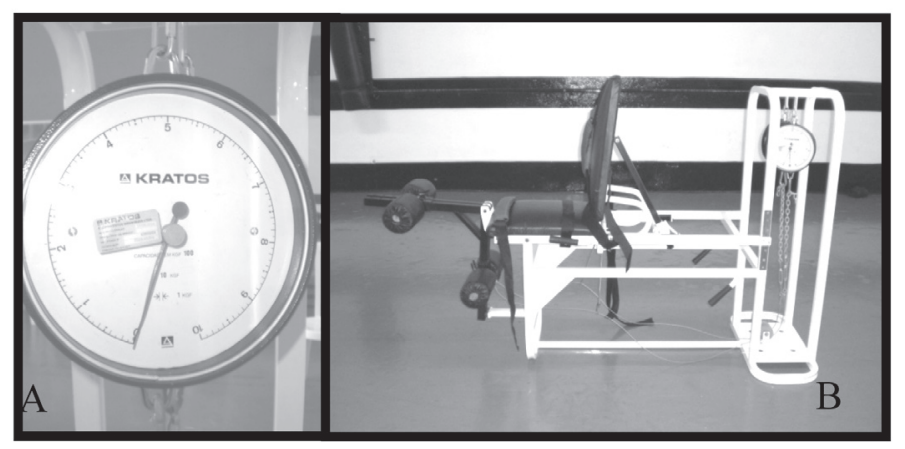

Figura 1 - (A) Dinamômetro analógico de tração Kratos ${ }^{\circledR}$. (B) Equipamento de mecanoterapia adaptado para acomodar o dinamômetro analógico Kratos $®$ na região posterior do equipamento.

Antes de cada avaliação do desempenho muscular do torque extensor e flexor isométrico foi realizada a calibração do equipamento. A calibração foi feita por um peso de $5 \mathrm{~kg}$ colocado primeiramente em uma balança digital Filizola ${ }^{\circledR}$ para confirmação do peso e em seguida conectado aos dois dinamômetros Kratos $^{\circledR}$ para confirmação da carga. O tronco, quadril e a coxa dos voluntários foram estabilizados com cintas de velcro (Figura 2a.). O suporte de resistência distal ou apoio tibial foi colocado dois dedos acima do maléolo lateral. Os voluntários foram instruídos a segurar seus braços cruzados sobre o tórax para isolar o momento extensor do joelho. Após o posicionamento correto do voluntário no equipamento DIM foi realizado o posicionamento do joelho na angulação de $60^{\circ}$ de flexão. Para a avaliação do torque flexor isométrico, o paciente foi posicionado em decúbito ventral e cintos de velcro foram colocados para estabilização da cintura pélvica para evitar compensações. Após o posicionamento correto do voluntário, foi realizado o posicionamento do joelho na angulação de $30^{\circ}$ de flexão. O paciente foi instruído a flexionar lentamente o joelho da posição de repouso, aproximadamente $0^{\circ}$, até que se crie tensão no cabo de aço bloqueando a continuação do movimento; quando o bloqueio era detectado, o avaliador posicionado ao lado do paciente utilizava o goniômetro para mensurar a angulação do joelho (Figura 2b). Após o correto posicionamento dos voluntários, foi realizada a familiarização do procedimento. Primeiramente, foi requisitado ao paciente que, após o comando verbal "preparar", ele estendesse (para verificação do torque extensor) ou flexionasse (para verificação do torque flexor) lentamente o joelho até criar novamente tensão no cabo de aço; após a verificação de que o movimento de extensão estava bloqueado, era requisitada força máxima de extensão do joelho durante cinco segundos sob comando verbal constante do examinador. Foi realizada uma repetição de familiarização, em que o participante exerceu $100 \%$ de sua força voluntária, seguida de mais três contrações isométricas voluntárias máximas (CIVM) com duração de cinco segundos e intervalos entre repetições de 90 segundos. O exame foi iniciado com a perna não dominante no grupo $\mathrm{GC}$ e na perna contralateral nos grupos GTP e GTF. Todos os valores dados em kgf foram anotados, desprezando-se apenas o valor referente à tentativa de familiarização.

Para efeito de validação do DIM na avaliação do desempenho muscular, os mesmos voluntários realizaram a segunda etapa de testes do torque extensor e flexor do joelho com intervalo de uma semana no dinamômetro isocinético computadorizado (Biodex Multi-Joint System 3 Pro) pertencente ao Laboratório de Avaliação e Intervenção em Ortopedia e Traumatologia (LAIOT) do Curso de Pós- Graduação em Fisioterapia da Universidade Federal de São Carlos (UFScar), configurado previamente através de seu software específico para operar no modo isométrico e isocinético.

Antes de iniciar os testes, foi realizada a randomização dos testes isométricos e isocinéticos através de sorteio.

Após a calibração do equipamento, os voluntários realizaram aquecimento em uma bicicleta ergometria por cinco minutos e alongamentos para o quadríceps e os isquiotibiais. Após o aquecimento, os pacientes foram posicionados na cadeira do dinamômetro isocinético e estabilizados no tronco, pelve e quadril por cintas de contenção para evitar compensações durante o exame (Figura 2c). 


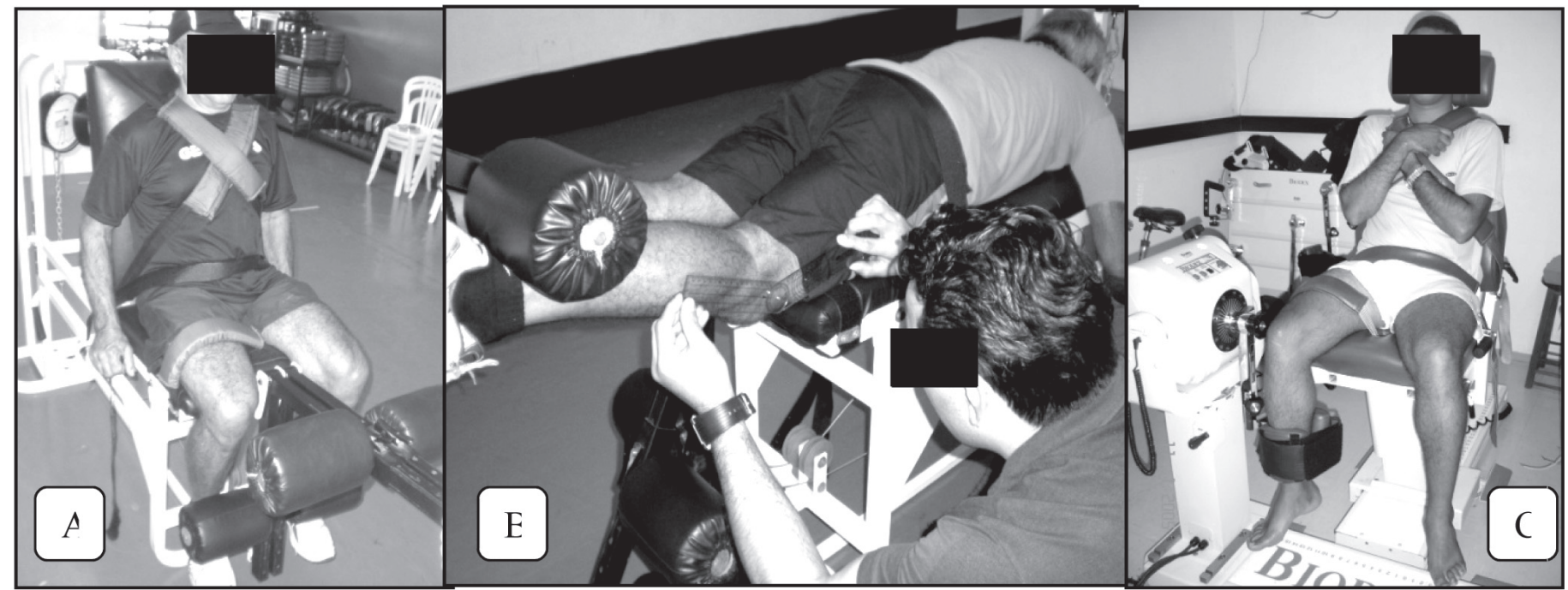

Figura 2 - (A) Posicionamento dos voluntários para coleta do torque extensor isométrico no dinamômetro isométrico modificado (DIM). (B) Posicionamento dos voluntários para coleta do torque flexor isométrico no DIM. (C) Posicionamento dos voluntários para coleta do torque extensor e flexor isométrico e isocinético do Biodex System 3.

Para a avaliação do torque extensor isométrico, o joelho foi posicionado no ângulo de $60^{\circ}$ de flexão. Após o posicionamento do joelho, foram dadas orientações ao paciente de realizar CIVM de extensão do joelho por cinco segundos com intervalos de 90 segundos entre as contrações. A primeira tentativa foi realizada para familiarização do voluntário seguindo três CIVM. Para a avaliação do torque flexor o joelho foi posicionado no ângulo de $30^{\circ}$ de flexão. Após o posicionamento do joelho, os voluntários realizaram CIVM de flexão do joelho por cinco segundos com intervalos de 90 segundos entre as contrações, seguindo idêntico protocolo para obtenção do torque extensor isométrico. Para a avaliação do torque isocinético, foi elaborado protocolo com duas velocidades $60^{\circ} / \mathrm{s}$ e $180^{\circ} / \mathrm{s}$. Na velocidade de $60 \%$ s os voluntários realizaram duas séries de cinco movimentos de extensão e flexão do joelho; na velocidade de $180 \%$ s, duas séries de cinco e 15 repetições. Em ambas as velocidades os voluntários realizaram movimentos entre os ângulos de 0 e 90 graus o mais rápido e forte possível, com intervalos entre séries de 90 segundos. A primeira série foi utilizada para familiarização do paciente com o teste isocinético.

O exame para todos os testes foi iniciado com a perna não dominante no grupo GC e na perna contralateral no grupo GTP e GTF.

\section{ANÁLISE ESTATÍSTICA:}

\section{Teste de confiabilidade intraexaminador}

A confiabilidade intraexaminador do DIM foi realizada previamente ao inicio dos testes isométricos e isocinéticos.
Foram convidados através de convite verbal nove voluntários sem lesões no joelho a participar do estudo. Os nove voluntários realizaram protocolo completo de avaliação do torque flexor e extensor isométrico do joelho descrito previamente neste trabalho. O protocolo de teste foi repetido dentro de 24 horas após o termino da primeira sequência de teste. A diferença em relação ao teste normal é que um examinador independente foi responsável pela coleta dos dados em kgf, sendo o examinador principal impedido de ver os resultados na primeira e segunda sequência de testes. Foi calculado o ICC interdias entre o maior valor obtido no torque extensor e flexor entre a primeira e segunda sequências de testes. Para realizar o estudo da confiabilidade intraexaminador teste-reteste do DIM, foi utilizado o coeficiente de correlação intraclasse ICC para a variação das medidas interdias.

O equipamento de DIM não apresenta uma interface com o computador e, consequentemente, com software especifico para cálculo dos déficits. Portanto, os dados referentes ao torque isométrico foram anotados na ficha de avaliação dos voluntários e posteriormente calculados utilizando a seguinte equação:

$$
\begin{gathered}
\text { Deficit }=\frac{\text { lado não dominante }}{\text { lado dominante }}(\times \text { 100) }(-100) \\
\text { GRUPO GC }
\end{gathered}
$$

$$
\text { Deficit }=\frac{\text { joelho lesado }}{\text { joelho contralateral }}(\times 100)(-100)
$$

GRUPO GTP e GTF 
Validade do DIM na avaliação do desempenho muscular dos extensores e flexores do joelho

Para comparar as medidas entre si dentro de cada grupo foi utilizado o método de análise de variância (ANOVA) para medidas repetidas, ou seja, comparar dentro de cada grupo o mesmo individuo realizando o teste isométrico no DIM, isométrico no Biodex System 3 , isocinético a $60 \%$ e $180 \%$ s no Biodex System 3 para o grupo dos extensores e flexores do joelho, com o intuito de analisar se há diferenças entre os 4 tipos de testes.

O segundo método de analise da validade foi o cálculo da sensibilidade e especificidade do DIM em detectar déficits significativos ou déficits dentro dos parâmetros normais. Déficits significativos foram definidos como diferenças $>15 \%$ entre joelhos e déficit normal valores $\leq 15 \%{ }^{(19)}$. O critério de sensibilidade demonstra o quanto um teste positivo é sensível para detectar a doença ou, no caso do presente estudo, o déficit muscular significativo definido como $>15 \%$ de diferença entre joelhos ${ }^{(20)}$. O critério de especificidade demonstra o quanto um teste negativo é especifico para detectar nenhuma doença ou, no caso do presente estudo, déficit dentro dos parâmetros normais $\leq 15 \%{ }^{(20)}$. O DIM foi comparado com os três testes realizados no Biodex System 3 no modo isométrico e isocinético a $60 \%$ e $180^{\circ} / \mathrm{s}$ de velocidade, considerando esses testes como padrão ouro.

Foi utilizado também o cálculo de concordância Kappa entre o DIM e os três tipos de testes de força realizados no Biodex System 3 pelos três grupos de voluntários. A classificação da intensidade da concordância entre os testes foi baseada no valor de Kappa, sendo: (concordância excelente: $>0,75)$, (concordância de moderada-forte: $r=$ 0,40-0,75), (concordância pobre: $<0,40)^{(21)}$. Para a análise de concordância Kappa os três grupos foram resumidos a apenas um grupo de 60 sujeitos e comparando o DIM com Biodex isométrico, o DIM x Biodex isocinético a $60 \%$ e DIM x Biodex isocinético a $180 \%$ s. A análise foi realizada para ambos os grupos musculares (extensores e flexores).

\section{Comparação intergrupos do torque isométrico dos extensores e flexores do joelho}

Para realizar a comparação entre os grupos GC, GTP e GTF nos testes isométricos, isocinéticos, questionário de Lysholm e artrometria, foi utilizada análise de variância (ANOVA) com teste post hoc de Bonferroni tendo como valor de $\mathrm{p}<0,05$.

\section{RESULTADOS}

$\mathrm{Na}$ tabela 1 podemos observar as características dos três grupos nos itens relacionados idade, peso, altura,
ADM de flexão e extensão, perimetria de coxa, nível de participação esportiva através da escala de Tegner ${ }^{(18)}$, tempo de pós-operatório e tipo de reabilitação utilizada no pós-operatório nos grupos GTP e GTF. Podem-se observar, também nas tabelas 1 e 2, ausência de diferenças significativas na ADM de flexão e extensão e frouxidão ligamentar pós-operatória demonstrando sinais de técnica cirúrgica adequada em ambos os grupos.

$\mathrm{Na}$ tabela 2 pode-se observar a diferença entre os grupos GC, GTP e GTF em relação à frouxidão ligamentar e questionário de Lysholm. Na avaliação da frouxidão ligamentar através do KT 1000 foi observada diferença entre joelhos no valor de translação anterior da tíbia significativamente menor no grupo GC em comparação com os grupos GTP e GTF $(\mathrm{p}<0,05)$, porém, nos três grupos a média de frouxidão ligamentar ficou dentro dos parâmetros normais abaixo de $3 \mathrm{~mm}$. Não houve diferença significativa entre os grupos GTP e GTF. No questionário de Lysholm utilizado para caracterização da amostra, ambos os grupos apresentaram valores menores que os do grupo controle, porém, o grupo GTP demonstrou pontuação significativamente menor em relação ao grupo GTF $(\mathrm{p}<0,05)$.

Tabela 2 - Comparação entre grupos dos valores de frouxidão ligamentar entre joelhos através do KT 1000 e pontuação obtida no questionário de Lysholm utilizando-se de análise de variância ANOVA com teste post hoc de Bonferroni $(p<0,05)$

\begin{tabular}{|c|c|c|c|c|c|c|}
\hline Teste & $\begin{array}{c}\text { Grupo } \\
\text { controle } \\
N=20\end{array}$ & (SD) & $\begin{array}{c}\text { Grupo } \\
\text { GTP } \\
N=20\end{array}$ & (SD) & $\begin{array}{c}\text { Grupo } \\
\text { GTF } \\
N=20\end{array}$ & (SD) \\
\hline $\begin{array}{l}\text { Questionário } \\
\text { de Lysholm }\end{array}$ & $100^{*}$ & 0 & $87,4^{* *}$ & 12,9 & 93,8 & 6,14 \\
\hline KT 1000 (mm) & $0,7 \dagger$ & ,49 & 2,94 & 2,26 & 2,89 & 1,98 \\
\hline \multicolumn{7}{|c|}{$\begin{array}{l}\text { Legendas: GC = grupo controle, GTP = grupo tendão patelar, GTF = grupo tendões flexores } \\
\text { * Grupo GC com pontuação significativamente maior que a do grupo GTP e GTF }(p<0,001) \\
\text { ** Grupo GTP com pontuação significativamente menor que a do grupo GTF }(p<0,001) \\
\text { † Grupo GC com valores da diferença de translação anterior da tíbia entre joelhos significativa- } \\
\text { mente menores que os do grupo GTP e GTF }(p<0,001)\end{array}$} \\
\hline \multicolumn{7}{|c|}{ Fonte: FMRP-USP } \\
\hline
\end{tabular}

Confiabilidade intraexaminador teste-reteste no DIM

O DIM demonstrou excelente confiabilidade intraexaminador teste-reteste em um intervalo de $24 \mathrm{~h}$, atingindo valores de ICC de 0,95, IC 95\% (0,87-0,98) para torque extensor e valores de ICC de 0,95 , IC 95\% $(0,87-0,98)$ para torque flexor (Tabela 3$)$. Foi realizado também o teste $t$ de Student para analisar a diferença interdias das médias do torque extensor e flexor do joelho. Os resultados demonstraram não haver diferenças significativas interdias para o torque extensor e flexor do joelho (Tabela 3). 
Tabela 3 - Teste de confiabilidade intraexaminador, ICC teste-reteste para avaliação do déficit extensor e flexor joelho coletado em nove indivíduos normais (18 joelhos) interdias com 24 horas de intervalo no dinamômetro isométrico modificado (DIM).

\begin{tabular}{c|c|c|c|c|c}
\hline $\mathbf{N}$ & $\begin{array}{c}\text { Grupo } \\
\text { muscular }\end{array}$ & $\begin{array}{c}\text { Média 1ㅇ } \\
\text { teste }\end{array}$ & $\begin{array}{c}\text { Média 2 } \\
\text { teste }\end{array}$ & $\begin{array}{c}\text { ICC }{ }^{2,1} \\
\text { interdias }\end{array}$ & IC 95\% \\
\hline $\begin{array}{c}9 \text { indivíduos } \\
\text { (18 joelhos) }\end{array}$ & Extensor & $49,7 \pm 10,7$ & $49,8 \pm 11,6$ & 0,95 & $(0,87-0,98)$ \\
\hline $\begin{array}{l}9 \text { indivíduos } \\
\text { (18 joelhos) }\end{array}$ & Flexor & $18,9 \pm 5,41$ & $19,06 \pm 5,12$ & 0,95 & $(0,87-0,98)$ \\
\hline \multicolumn{7}{l|l}{ Fonte: FMRP-USP }
\end{tabular}

\section{Comparação do déficit do torque extensor intragru- pos e intergrupos}

Quando a comparação feita intragrupos, não houve diferenças significativas na média dos déficits de torque extensor nas quatro modalidades de teste nos grupos GC e GTF (Tabela 4). No grupo GTP os valores médios de déficits do torque extensor foram significativamente maiores no teste isométrico realizado no DIM e no isocinético a $60 \%$ s no Biodex System 3, quando comparados com os testes isométrico e isocinético a $180 \%$, ambos realizados no Biodex System 3 ( $\mathrm{p}<0,001)$ (Tabela 4).

Tabela 4 - Comparação dos déficits do torque extensor e flexor entre grupos coletados no dinamômetro isométrico modificado (DIM), Biodex modo isométrico, isocinético $600 / \mathrm{s}$ e $180 \%$ s. Análise de variância ANOVA com teste post hoc de Bonferroni

\begin{tabular}{c|c|c|c|c|c|c}
\hline Teste & $\begin{array}{c}\text { Grupo GC } \\
\mathrm{N}=20\end{array}$ & $(\mathrm{SD})$ & $\begin{array}{c}\text { Grupo } \\
\mathrm{GTP} \\
\mathrm{N}=20\end{array}$ & $(\mathrm{SD})$ & $\begin{array}{c}\text { Grupo } \\
\mathrm{GTF} \\
\mathrm{N}=20\end{array}$ & $(\mathrm{SD})$ \\
\hline DIM (extensores) & $6,81^{*}$ & $(4,59)$ & 32,11 & $(15,24)$ & $18,69^{* *}$ & $(18,90)$ \\
\hline $\begin{array}{c}\text { Isométrico Biodex } \\
\text { (extensores) }\end{array}$ & $8,15^{\star}$ & $(4,63)$ & 25,45 & $(11,65)$ & 18,23 & $(15,42)$ \\
\hline $\begin{array}{c}\text { Isocinético 60\%/s } \\
\text { (extensores) }\end{array}$ & $7,71^{*}$ & $(4,21)$ & 32,30 & $(14,30)$ & $18,70^{\star *}$ & $(17,79)$ \\
\hline $\begin{array}{c}\text { Isocinético 180\%/s } \\
\text { (extensores) }\end{array}$ & $5,85^{\star}$ & $(3,89)$ & 22,63 & $(10,51)$ & 15,83 & $(13,26)$ \\
\hline $\begin{array}{c}\text { DIM (flexores) } \\
\text { Isométrico }\end{array}$ & 8,27 & $(7,27)$ & $-1,42^{* * *}$ & $(13,96)$ & 8,38 & $(16,34)$ \\
\hline $\begin{array}{c}\text { Biodex(flexores) } \\
\text { Isocinético 60\% } \\
\text { (flexores) }\end{array}$ & 5,28 & $(5,44)$ & 4,64 & $(9,84)$ & 8,90 & $(7,25)$ \\
\hline $\begin{array}{c}\text { Isocinético 180\%/s } \\
\text { (flexores) }\end{array}$ & 6,09 & $(3,85)$ & 2,51 & $(13,49)$ & 7,20 & $(9,85)$ \\
\hline
\end{tabular}

Legenda: $\mathrm{GC}=$ grupo controle, GTP = grupo tendão patelar, GTF = grupo tendões flexores * Grupo controle com déficit no torque extensor significativamente menor que o do grupo GTP e GTF $(p<0,001){ }^{-* \star}$ Grupo GTF com déficit significativamente menor no torque extensor que o do grupo GTP $(p<0,001)$ - *** Grupo GTP com déficit significativamente menor no torque flexor que o do grupo GC e GTF $(p<0,001)$

Fonte: FMRP-USP
Quando a comparação é feita intergrupos, valores de déficit no torque extensor foram significativamente menores no grupo GC nas quatro modalidades de testes realizados comparados com os grupos GTP e GTF $(\mathrm{p}<0,001)$ (Tabela 4).

O grupo GTF demonstrou déficits significativamente menores de torque extensor comparados com o GTP no teste isométrico realizado no DIM e no isocinético a $60 \%$ s no Biodex System 3 ( $p<0,001$ ), não sendo observadas diferenças entre GTP e GTF nos testes isométrico e isocinético a $180 \%$ s, ambos realizados no Biodex System 3 (Tabela 4).

\section{Comparação do déficit do torque flexor intragrupos e intergrupos}

$\mathrm{Na}$ análise intragrupos, apenas o grupo GTP demonstrou valores de déficits significativamente menores no teste isométrico realizado no DIM em comparação com as demais modalidades de teste Biodex isométrico, Biodex isocinético a $60 \%$ s e Biodex isocinético a $180 \% \mathrm{~s}$ $(\mathrm{p}<0,001)$ Tabela 5). Na análise intergrupos, o grupo GTP demonstrou déficit significativamente menor que o dos grupos GC e GTF apenas no teste isométrico realizado no DIM, demonstrando valores negativos, sinal de que a média de torque flexor desenvolvido pelo membro lesado foi maior ou sobrepôs os valores do membro contralateral saudável $(\mathrm{p}<0,001)$ (Tabela 4).

\section{Sensibilidade e especificidade do DIM na avaliação de déficits no torque extensor}

No grupo GC foram observados valores de $0 \%$ a $50 \%$ de sensibilidade e entre $94,7 \%$ e $100 \%$ de especificidade na comparação entre o teste realizado no DIM e tendo como padrão ouro o teste isométrico e o isocinético a $60 \%$ s e $180^{\circ} / \mathrm{s}$ realizado no Biodex System 3 (Tabela 5). No grupo GTP foram observados valores de $86,7 \%$ a $89,5 \%$ de sensibilidade e $20 \%$ a $100 \%$ de especificidade (Tabela 5). No grupo GTF foram detectados valores entre $66,7 \%$ e $88,9 \%$ de sensibilidade e $54,6 \%$ a $72,7 \%$ de especificidade (Tabela 5).

\section{Sensibilidade e especificidade do DIM na avaliação de déficits no torque flexor}

No grupo GC foram observados valores de $0 \%$ de sensibilidade e $84,2 \%$ a $85 \%$ de especificidade na comparação entre o teste realizado no DIM e tendo como padrão ouro o teste isométrico e isocinético a $60^{\circ} \%$ s e $180 \%$ realizado no Biodex System 3 (Tabela 5). No grupo GTP foram observados valores de $33 \%$ a $50 \%$ de sensibilidade e $100 \%$ de especificidade (Tabela 5). No grupo GTF foram detectados valores entre $60 \%$ e $83,3 \%$ de sensibilidade e $58,8 \%$ a $71,4 \%$ de especificidade (Tabela 5). 
Tabela 5 - Sensibilidade e especificidade do dinamômetro isométrico modificado (DIM) para detectar sujeitos do grupo controle (GC), grupo tendão patelar (GTP) e grupo tendões flexores (GTF) com déficits significativos $>15 \%$ ou sem $<15 \%$ do torque extensor e flexor do joelho tendo como padrão ouro o testes isométrico e o isocinético realizado no Biodex system 3

\begin{tabular}{l|c|c|c|c}
\hline \multicolumn{1}{c|}{ Teste } & Sensibilidade & IC 95\% & Especificidade & IC 95\% \\
\hline DIM x BITE (GC) & $50 \%$ & $(1,3-98,7 \%)$ & $100 \%$ & $(81,5-98,7 \%)$ \\
\hline DIM x B60TE (GC) & $0 \%$ & $(0,0-97,5 \%)$ & $95 \%$ & $(74-97,5 \%)$ \\
\hline DIM x B180TE (GC) & $0 \%$ & $(0,0-97,5 \%)$ & $94,7 \%$ & $(74-99,9 \%)$ \\
\hline DIM x BITE (GTP) & $88,9 \%$ & $(65,3-98,6 \%)$ & $50 \%$ & $(1,3-98,7 \%)$ \\
\hline DIM x B60TE (GTP) & $89,5 \%$ & $(66,9-98,7 \%)$ & $100 \%$ & $(2,5-100 \%)$ \\
\hline DIM x B180TE (GTP) & $86,7 \%$ & $(59,6-98,3 \%)$ & $20 \%$ & $(1,0-71,7 \%)$ \\
\hline DIM x BITE (GTF) & $88,9 \%$ & $(51,7-99,7 \%)$ & $72,7 \%$ & $(39,1-94 \%)$ \\
\hline DIM x B60TE (GTF) & $77,8 \%$ & $(40-97,2 \%)$ & $63,6 \%$ & $(30,8-89,1 \%)$ \\
\hline DIM x B180TE (GTF) & $66,7 \%$ & $(29,9-92,5 \%)$ & $54,6 \%$ & $(23,4-83,3 \%)$ \\
\hline DIM x BITFLEX (GC) & $0 \%$ & $(0,0-97,5 \%)$ & $84,2 \%$ & $(60,4-96,6 \%)$ \\
\hline DIM x B60TFLEX (GC) & $0 \%$ & $(0,0-97,5 \%)$ & $84,2 \%$ & $(60,4-96,6 \%)$ \\
\hline DIM x B180TFLEX (GC) & $0 \%$ & $(0,0-97,5 \%)$ & $85 \%$ & $(60,4-96,6 \%)$ \\
\hline DIM x BITFLEX (GTP) & $33 \%$ & $(1-90,6 \%)$ & $100 \%$ & $(80,5-100 \%)$ \\
\hline DIM x B60TFLEX (GTP) & $50 \%$ & $(1,3-98,7 \%)$ & $100 \%$ & $(81,5-100 \%)$ \\
\hline DIM x B180TFLEX (GTP) & $50 \%$ & $(1,3-98,7 \%)$ & $100 \%$ & $(81,5-100 \%)$ \\
\hline DIM x BITFLEX (GTF) & $66,7 \%$ & $(9,4-99,2 \%)$ & $58,8 \%$ & $(33-81,6 \%)$ \\
\hline DIM x B60TFLEX (GTF) & $60 \%$ & $(14,7-94,7 \%)$ & $60 \%$ & $(32,2-83,7 \%)$ \\
\hline DIM x B180TFLEX (GTF) & $83,3 \%$ & $(35,9-99,6 \%)$ & $71,4 \%$ & $(41,9-91,6 \%)$ \\
\hline
\end{tabular}

Legenda: DIM = teste dinamômetro isométrico modificado, BITE = Biodex isométrico torque extensor, B60TE $=$ Biodex isocinético a $60 \%$ torque extensor, B180TE $=$ Biodex isocinético a $180 \%$ torque extensor, BITFLEX = Biodex isométrico torque flexor, B60TFLEX = Biodex isocinético a $60 \%$ torque flexor, B180TE = Biodex isocinético a $180 \%$ s torque flexor Fonte: FMRP-USP

Análise de concordância intertestes através do método Kappa para todos os grupos $(\mathrm{N}=60)$

$\mathrm{Na}$ análise de concordância para o torque extensor comparando o DIM com os três testes realizados no Biodex System 3, os valores de Kappa permaneceram entre 0,46 e 0,73 , demonstrando concordância moderada a forte (Tabela 6). Quando a comparação é feita somente entre os três testes realizados do Biodex System 3, os valores de Kappa permaneceram entre 0,55 e 0,63, obtendo a mesma classificação (Tabela 6).

$\mathrm{Na}$ análise de concordância para o torque flexor entre os testes, os valores de Kappa permaneceram entre 0,17 e 0,48, demonstrando concordância significativa na comparação entre o teste isométrico DIM e o isocinético a $180 \%$ $(\mathrm{k}=0,48 ; \mathrm{p}<0,001)$, (Tabela 6). Quando a comparação é feita somente entre os três testes realizados do Biodex System 3, os valores de Kappa permaneceram entre 0,42 e 0,56, demonstrando concordância moderada a forte (Tabela 6).
Tabela 6 - Análise de concordância intertestes através do método Kappa para todos os grupos a respeito dos déficits do torque extensor e flexor $(\mathrm{N}=60)$

\begin{tabular}{l|c|c|c}
\hline \multicolumn{1}{c|}{ Teste } & Kappa & p & IC 95\% \\
\hline DIM x Biodex iso (extensores) & 0,73 & $<0,001$ & $(0,48-0,98)$ \\
\hline DIM x Biodex 60\% (extensores) & 0,70 & $<0,001$ & $(0,44-0,95)$ \\
\hline DIM x Biodex 180\% (extensores) & 0,46 & $<0,001$ & $(0,21-0,71)$ \\
\hline Biodex iso x Biodex 60\% (extensores) & 0,63 & $<0,001$ & $(0,38-0,88)$ \\
\hline Biodex iso x Biodex 180\%s (extensores) & 0,56 & $<0,001$ & $(0,31-0,81)$ \\
\hline Biodex 60\%/s x Biodex 180\% & 0,55 & $<0,001$ & $(0,30-0,81)$ \\
\hline (extensores) & 0,25 & 0,037 & $(0,01-0,50)$ \\
\hline DIM x Biodex iso (flexores) & 0,25 & 0,037 & $(0,01-0,50)$ \\
\hline DIM x Biodex iso (flexores) & 0,17 & $<0,148$ & $(-0,06-0,41)$ \\
\hline DIM x Biodex 60\% (flexores) & 0,48 & $<0,001$ & $(0,24-0,72)$ \\
\hline DIM x Biodex 180\% (flexores) & 0,46 & $<0,001$ & $(0,21-0,71)$ \\
\hline Biodex iso x Biodex 60\% (flexores) & 0,56 & $<0,001$ & $(0,31-0,82)$ \\
\hline Biodex iso x Biodex 180\%s (flexores) & 0,42 & $=0,001$ & $(0,17-0,67)$ \\
\hline Biodex 60\%/s x Biodex 180\% (flexores) &
\end{tabular}

Legenda: $\mathrm{DIM}=$ dinamômetro isométrico modificado - Biodex iso = teste isométrico realizado no dinamômetro Biodex system $3-$ Biodex $60 \%$ s = teste isocinético realizado a $60 \%$ s no dinamômetro Biodex system 3 - Biodex $180 \%$ s = teste isocinético realizado a $180 \%$ no dinamômetro Biodex system 3

Fonte: FMRP-USP

\section{DISCUSSÃO}

O primeiro objetivo do estudo foi analisar a confiabilidade intraexaminador teste-reteste do DIM. Os resultados demonstraram excelente confiabilidade intraexaminador na avaliação do torque extensor e flexor do joelho em indivíduos saudáveis com ICC de 0,95 para os extensores e flexores do joelho.

Esses valores de coeficiente de correlação intraclasse são muito semelhantes aos de trabalhos que utilizaram diferentes dinamômetros para avaliar desempenho muscular em diferentes articulações e indivíduos ${ }^{(8-10)}$.

Outro objetivo do trabalho foi analisar diretamente se os déficits coletados no equipamento DIM seriam semelhantes aos déficits encontrados no equipamento Biodex System 3 operando no modo isométrico e isocinético. Para isso foram utilizados os testes de sensibilidade e especificidade e cálculo da concordância entre medidas utilizando-se do coeficiente Kappa. Esses métodos foram utilizados devido a seu uso frequente em epidemiologia, principalmente para testar métodos e técnicas de diagnóstico ${ }^{(20,21)}$. Como o principal objetivo de utilizar dinamômetros isométricos e isocinéticos é quantificar e definir desequilíbrios e anormalidades do sistema musculoesquelético, o ideal seria investigar a concordância entre dois equipamentos em fornecer dados semelhantes. 
Os resultados demonstraram que o DIM demonstrou de boa a alta especificidade na avaliação dos extensores e flexores do joelho no grupo GC comparado com os testes isométrico e isocinético no Biodex System 3, alta especificidade na avaliação dos extensores e flexores do joelho comparado com o teste isocinético a $60^{\circ} \mathrm{s}$. No grupo GTP, o DIM demonstrou valores razoáveis de especificidade na avaliação dos extensores e flexores do joelho; no entanto, mostrou boa sensibilidade na avaliação dos extensores do joelho no grupo GTP. No grupo GTF, boa sensibilidade foi observada na avaliação nos extensores comparada com a do teste isométrico no Biodex System 3; na comparação com os demais testes houve razoável sensibilidade, com valores permanecendo entre $66,7 \%$ e $77,8 \%$. Na avaliação dos flexores do joelho no grupo GTF, o DIM demonstrou boa sensibilidade comparada com a do teste isocinético a $180^{\circ}$ /s; na comparação com os demais testes, os valores de sensibilidade foram razoáveis, permanecendo entre $60 \%$ e $67 \%$. Não foram encontrados na literatura trabalhos que comparassem dois métodos de mensuração de déficits musculares utilizando critérios de sensibilidade e especificidade.

Na avaliação do coeficiente de concordância Kappa, foram demonstrados valores de moderado a forte de concordância $(k=0,43-0,73 ; p<0,001)$ entre o DIM e os testes isométricos e isocinéticos realizados no Biodex System 3 na avaliação do torque extensor.

Esses valores de concordância são semelhantes aos encontrados por Martin et al $(\mathrm{k}=0,69 ; \mathrm{p}<0,001)$, que utilizaram o coeficiente Kappa para comparação entre o dinamômetro digital manual e o Biodex System 3 utilizando somente contrações isométricas em indivíduos idosos ${ }^{(13)}$.

$\mathrm{Na}$ avaliação do torque flexor, o índice de concordância Kappa demonstrou valores de moderado a forte somente na comparação do DIM com o teste isocinético a $180^{\circ}$ /s e pobre concordância quando o DIM foi comparado com o teste isométrico e isocinético do Biodex System 3, respectivamente.

Alguns autores acreditam que o coeficiente Kappa nem sempre demonstra um resultado justo, pois apresenta valores baixos mesmo havendo alta concordância entre julgamentos ${ }^{(21,22)}$.

Björklund et al analisaram a concordância na avaliação de desempenho nas lesões do joelho em atletas entre dois fisioterapeutas. Em um dos testes utilizados o coeficiente de Kappa mostrou consistência moderadaforte de concordância $(\mathrm{k}=0,54)$, embora a concordância entre os dois fisioterapeutas fosse de $85 \%{ }^{(22)}$. No presente trabalho foi observado o mesmo comportamento na avaliação do torque flexor no grupo GTF, comparan- do o DIM com o teste isométrico e isocinético a $60 \%$ no Biodex; os valores de Kappa permaneceram baixos (0,17-0,25), porém, os dois equipamentos classificaram igualmente em déficit presente ou ausente $76 \%$ e $78 \%$ dos 60 indivíduos, respectivamente.

$\mathrm{Na}$ analise intergrupos, maiores déficits de torque extensor foram encontrados no grupo GTP em comparação com o grupo GTF em todos os quatro testes realizados, porém, com valor de $p$ estatisticamente significativo somente em dois testes: DIM isométrico $(32,11 \pm 15,24)$ e isocinético a $60^{\circ}$ /s $(32,30 \pm 14,30)$. Esses resultados são similares aos de trabalhos que demonstraram maiores déficits no torque extensor em pacientes com enxerto do tendão patelar ${ }^{(23,24)}$. Os valores médios de déficits encontrados nos grupos GTP e GTF são similares aos encontrados em trabalhos que analisaram déficits do torque extensor no período de seis a sete meses de pós-operatório, com valores médios de déficit permanecendo entre $24.6 \%$ e $37 \%$ para técnicas de enxerto do tendão patelar e entre $21,6 \%$ e $26 \%$ para técnicas de enxerto dos tendões flexores ${ }^{(25,26)}$

$\mathrm{Na}$ análise do torque flexor intergrupos pode-se observar de maneira geral que o grupo GTF apresentou média de déficits maior que a do GTP em todos os quatro testes; no entanto,o valor de $p$ foi estatisticamente significativo apenas no teste realizado no DIM $(8,38 \pm 16,34$ contra $-1,42 \pm 13,96$, respectivamente). Os valores médios de déficits encontrados nos grupos GTP e GTF são similares aos de trabalhos que analisaram déficits do torque flexor no pós-operatório, com valores médios de déficit permanecendo entre -1 e $6 \%$ para técnicas de enxerto do tendão patelar e entre 3\% e 10\% para técnicas de enxerto dos tendões flexores ${ }^{(26-28)}$. Não houve diferenças entre os grupos GTF e GC em todos os testes realizados.

Quando observamos o grupo GTF mais detalhadamente, 45\% (9/20) dos indivíduos do grupo GTF apresentaram déficits significativos $(>15 \%)$ no teste realizado no DIM; quando o teste foi realizado no dinamômetro Biodex System 3 no modo isométrico e isocinético a $60 \%$ s e $180^{\circ} / \mathrm{s}$, a percentagem de indivíduos com déficits significativos se reduz para 15\% (3/20), 20\% $(4 / 20)$ e $25 \%$ (5/20), respectivamente. Esses achados demonstram que a análise do torque flexor em decúbito ventral pode desafiar mais intensamente o individuo e os déficits podem ficar mais evidentes.

As variações encontradas no estudo a respeito do grupo GTF na avaliação do torque flexor podem ter relação com o posicionamento dos voluntários. Como pode ser visto na figura $2 b$, os voluntários são posicionados em decúbito ventral e estabilizados ao nível da cintura pélvica para avaliar o grupo dos flexores do joelho no DIM. 
No dinamômetro isocinético, os voluntários são posicionados na posição sentada com estabilização no tórax e cintura pélvica; é realizada a correção da gravidade para mensurar os valores de torque final (Figura 2c). No DIM não há correção da gravidade para a coleta dos dados. Para realizar contração isométrica máxima, os voluntários o fazem contra a gravidade e também contra o peso do braço de alavanca (Figura 2c). No Biodex System 3 a direção da tensão isométrica ou isocinética é na mesma direção da gravidade; portanto, mesmo padronizando o ângulo de $30^{\circ}$ para posicionamento do joelho para o teste do torque flexor isométrico, os valores podem variar entre modalidades e equipamentos. As variações dos valores coletados não ficam restringidas apenas ao DIM; dentro dos próprios testes realizados somente no Biodex System 3 , principalmente para coleta do torque flexor, os valores de concordância entre testes fornecidos pelo coeficiente Kappa permaneceram entre 0,42 e 0,56, ou seja, valores de moderado a forte de concordância (Tabela 1). A concordância entre os déficits encontrados entre as duas velocidades isocinéticos, $60 \% \mathrm{~s}$ e $180 \% \mathrm{~s}$ foi moderada a forte $(\mathrm{k}=0,42 ; \mathrm{p}<0,001)$, porém, abaixo dos valores de concordância entre o DIM e o teste isocinético a $180^{\circ}$ /s $(\mathrm{k}=0,48 ; \mathrm{p}<0,001)$. Esses resultados estão de acordo com estudos que investigaram a confiabilidade dos dinamômetros isocinéticos, sendo observada excelente confiabilidade teste-reteste para avaliação do pico de torque realizada no mesmo equipamento ${ }^{(29)}$, porém ocorrendo variabilidade mesmo em comparações entre dois dinamômetros isocinéticos de fabricantes diferentes ${ }^{(30)}$.

$\mathrm{Na}$ avaliação da frouxidão ligamentar intergrupos, $\mathrm{o}$ GC apresentou diferenças menores de frouxidão entre joelhos em comparação com o GTP e o GTF; porém, os valores médios de ambos os grupos permaneceram dentro dos padrões de normalidade $(3 \mathrm{~mm})^{(31)}$. Na avaliação através da escala de Lysholm, as pontuações médias dos grupos GTP e GTF permaneceram dentro de valores similares aos encontrados por outros estudos, variando entre 85 e 95 pontos para enxerto do tendão patelar e entre 86 e 92 para enxerto dos tendões flexores ${ }^{(32,33)}$.

Como o presente estudo teve como objetivo principal avaliar a validade do DIM dentro dos três grupos de indivíduos, as conclusões a respeito das diferenças entre os tipos de técnicas cirúrgicas relacionadas com o desempenho muscular ficam limitadas devido ao formato do estudo ser retrospectivo.

O DIM foi elaborado devido à dificuldade de acesso aos dinamômetros isocinéticos computadorizados. A adaptação de células de carga ou dinamômetros analógicos em equipamentos de musculação como utilizados neste estudo pode ser uma alternativa de baixo custo para avaliar déficits musculares nos membros inferiores, principalmente na articulação do joelho, desde que os avaliadores sigam protocolos rígidos para a coleta dos dados. Todos os critérios relacionados a técnicas para avaliação precisa da força e potência muscular recomendados pela American Society of Sports Physiology foram seguidos neste estudo para analisar a confiabilidade e validade do DIM, incluindo padronização do aquecimento, familiarização, ângulo articular, duração das contrações, comando verbal, intervalo de descanso, número de repetições, posicionamento e estabilização do voluntário ${ }^{(34)}$.

Este estudo demonstrou que, apesar das diferenças biomecânicas a respeito de testes isométricos e isocinéticos, ambos podem fornecer valores similares de déficits de força muscular.

Sapega define força muscular como a capacidade do músculo de gerar ativamente tensão, independente da condição especifica no qual esta tensão esteja sendo mensurada (velocidade lenta ou rápida de contração, contrações em estado de encurtamento ou alongamento); ele conclui que nenhum modo específico de teste (isométrico, isotônico ou isocinético) é considerado o melhor ou o mais válido para mensuração da força muscular ${ }^{(35)}$.

Essa afirmação de Sapega reflete de certo modo o abandono dos testes isométricos na década de 70 devido à criação e consequentemente utilização em larga escala dos dinamômetros isocinéticos nos Estados Unidos ${ }^{(35)}$. Recentemente, houve grande aumento de estudos utilizando novamente testes isométricos para avaliação do joelho em diferentes grupos de pacientes, patologias e intervenções ${ }^{(36,37)}$. No entanto, todos esses estudos utilizaram o modo isométrico no próprio equipamento isocinético, permanecendo então uma alternativa de alto custo e pouco acessível.

\section{CONCLUSÕES}

Este estudo, portanto, demonstrou que a adaptação de dinamômetros isométricos analógicos conectados a equipamentos de musculação é uma alternativa de fácil reprodução, com possibilidade de obter dados com alta confiabilidade intraexaminador teste-reteste e considerável validade na detecção de déficits musculares em indivíduos saudáveis e indivíduos submetidos à reconstrução do LCA. As variabilidades encontradas entre testes isométricos comparados com testes isocinéticos foram menores na avaliação do torque extensor do joelho. A avaliação do torque flexor isométrico em decúbito ventral pode evidenciar maiores déficits do torque flexor quando comparado com a posição sentada. 


\section{REFERÊNCIAS}

1. Lephart SM, Kocher MS, Harner CD, Fu FH. Quadriceps strength and functional capacity after anterior cruciate ligament reconstruction. Patellar tendon autograft versus allograft. Am J Sports Med. 1993;21(5):738-43.

2. Harilainen A, Sandelin J, Jansson KA. Cross-pin femoral fixation versus metal interference screw fixation in anterior cruciate ligament reconstruction with hamstring tendons: results of a controlled prospective randomized study with 2-year follow-up. Arthroscopy. 2005;21(1):25-33.

3. Grant JA, Mohtadi NG, Maitland ME, Zernicke RF. Comparison of home versus physical therapy-supervised rehabilitation programs after anterior cruciate ligament reconstruction: a randomized clinical trial. Am J Sports Med. 2005;33(9):1288-97.

4. Gobbi A, Domzalski M, Pascual J. Comparison of anterior cruciate ligament reconstruction in male and female athletes using the patellar tendon and hamstring autografts. Knee Surg Sports Traumatol Arthrosc. 2004;12(6):534-9.

5. Myer GD, Paterno MV, Ford KR, Quatman CE, Hewett TE. Rehabilitation after anterior cruciate ligament reconstruction: criteria-based progression through the return-to-sport phase. J Orthop Sports Phys Ther. 2006;36(6):385-402.

6. Magalhães J, Oliveira J, Ascensão A, Soares J. Concentric quadriceps and hamstrings isokinetic strength in volleyball and soccer players. J Sports Med Phys Fitness. 2004;44(2):119-25.

7. Dvir Z. Isocinética: Avaliações musculares, interpretações e aplicações clinicas. São Paulo: Manole; 2002. p.101-28.

8. Trudelle-Jackson E, Jackson AW, Frankowski CM, Long KM, Meske NB. Interdevice reliability and validity assessment of the Nicholas hand held dynamometer. J Orthop Sports Phys Ther. 1994;20(6):301-6.

9. Roy MA, Doherty TJ. Reliability of hand-held dynamometry in assessment of knee extensor strength after hip fracture. Am J Phys Med Rehabil. 2004;83(11):813-8.

10. Visser J, Mans E, de Visser M, Van der Berg-Vos Franssen $H$, de Jong JM. et al. Comparison of maximal voluntary isometric contraction and hand held dynamometry in measuring muscle strength of patients with progressive lower motor neuron syndrome. Neuromuscul Disord. 2003;13(9):744-50.

11. Kolber MJ, Beekhuizen K, Cheng MS, Fiebert IM. The reliability of hand-held dynamometry in measuring isometric strength of the shoulder internal and external rotator musculature using a stabilization device. Physiother Theory Pract. 2007;23(2):119-24.

12. May LA, Burnham RS, Steadward RD. Assessment of isokinetic and hand held dynamometer measures of shoulder rotator strength among individuals with spinal cord injury. Arch Phys Med Rehabil. 1997;78(3):251-5

13. Martin HJ, Yule V, Syddall HE, Dennison EM, Cooper C, Aihie Sayer A. Is handheld dynamometry useful for the measurement of quadriceps strength in older people? A comparison with the gold standard Biodex dynamometry. Gerontology. 2006;52(3):154-9.

14. Bohannon RW. Hand-held compared with isokinetic dynamometry for measurement of static knee extension torque (parallel reliability of dynamometers). Clin Phys Physiol Meas. 1990;11(3):217-22.

15. Reinking Mf, Bockrath-Pugliese K, Worrell T, Kegerreis RI, Miller-Sayers K, Farr J. Assessment of quadriceps muscle performance by hand-held, isometric, and isokinetic dynamometry in patients with knee dysfunction. J Orthop Sports Phys Ther. 1996;24(3):154-9.

16. Lysholm J, Gilquist J. Evaluation of knee ligament surgery results with special emphasis on use of a scoring Scale. Am J Sports Med. 1982;10(3):150-54

17. Peccin MS, Ciconelli R, Cohen M. Specific questionnaire for knee symptoms - the "Lysholm Knee Scoring Scale": translation and validation into Portuguese. Acta Ortop Bras. 2006;14(5):268-72.

18. Tegner Y, Lysholm J. Rating systems in the evaluation of knee ligament injuries. Clin Orthop Relat Res. 1985;(198):43-9.

19. Kvist J. Reabilitation following anterior cruciate ligament injury current recommendation for sports participation. Sports Med. 2004;34(4):269-80.
20. Karam FC, Silva JLB, Fridman MW, Abreu A, Arbor M, Abreu M, et al. A ressonância magnética para o diagnóstico das lesões condrais, meniscais e dos ligamentos cruzados do joelho. Radiol Bras. 2007;40(3):179-82.

21. Godinho GG, Freitas JMA, França FO, Lopes AA, Milazzo AJ, Zotto CD. Eficiência dos métodos de avaliação clínico (teste de O'Brien) e de imagem (artroressonância magnética) no diagnóstico das lesões SLAP no ombro. Rev Bras Ortop. 2006;41(11/12):461-6.

22. Björklund K, Sköld C, Andersson L, Dalén N. Reliability of a criterion-based test of athletes with knee injuries; where the physiotherapist and the patient independently and simultaneously assess the patient's performance. Knee Surg Sports Traumatol Arthrosc. 2006:14(2):165-75.

23. Moisala AS, Järvelä T, Kannus $P$, Järvinen M. Muscle Strength Evaluations after ACL Reconstruction. Int J Sports Med. 2007;28(10):868-72.

24. de Jong SN, van Caspel DR, van Haeff MJ, Saris DB. Functional assessment and muscle strength before and after reconstruction of chronic anterior cruciate ligament lesions. Arthroscopy. 2007;23(1):21-8.

25. Heijne A, Werner S. Early versus late start of open kinetic chain quadriceps exercises after ACL reconstruction with patellar tendon or hamstring grafts: a prospective randomized outcome study . Knee Surg Sports Traumatol Arthrosc. 2007;15(4):402-14

26. Jansson KA, Linko E, Sandelin J, Harilainen A. A prospective randomized study of patellar versus hamstring tendon autografts for anterior cruciate ligament reconstruction. Am J Sports Med. 2003;31(1):12-8.

27. Aune AK, Holm I, Risberg MA, Jensen HK, Steen H. Four-strand hamstring tendon autograft compared with patellar tendon-bone autograft for anterior cruciate ligament reconstruction. A randomized study with two-year follow-up. Am J Sports Med. 2001;29(6):722-8.

28. Anderson JL, Lamb SE, Barker KL, Davies S, Dodd CA, Beard DJ. Changes in muscle torque following anterior cruciate ligament reconstruction: acomparison between hamstrings and patella tendon graft procedures on 45 patients. Acta Orthop Scand. 2002;73(5):546-52.

29. Aitkens S, Lord J, Bernauer E, McCrory M. Analysis of the Validity of the lido digital isokinetic system. Phys Ther. 1987;67(4):756 -60.

30. Thompson MC, Shingleton LG, kegerreis ST. Comparisons of values generated during testing of the knee using the Cybex II plus $®$ and Biodex Model B-2000® isokinetic dynamometers. J Orthop Sports Phys Ther. 1989;11(2):108-15.

31. Barber-Westin SD, Noyes FR, Heckmann TP, Shaffer BL. The effect of exercise and rehabilitation on anterior-posterior knee displacements after anterior cruciate ligament autograft reconstruction. Am J Sports Med. 1999;27(1):84-93.

32. Ejerhed L, Kartus J, Sernert N, Kohler K, Karlsson J. Patellar tendon or semitendinosus tendon autografts for anterior cruciate ligament reconstruction? A prospective randomized study with a two-year follow-up. Am J Sports Med. 2003;31(1):19-25.

33. Shaieb MD, Kan DM, Chang SK, Marumoto JM, Richardson AB.Mark . A prospective randomized comparison of patellar tendon versus semitendinosus and gracilis tendon autografts for anterior cruciate ligament reconstruction. Am J Sports Med. 2002; 30(2):214-20.

34. Brown LE, Weir JP. Asep procedures recommendation i: accurate assessment of muscular strength and power. J Exp. Phys. 2001;4(1):1-21.

35. Sapega AA. Muscle performance evaluation in orthopaedic practice. J Bone Joint Surg Am. 1990;72(10):1562-74.

36. Holder-Powell HM, Di Matteo G, Rutherford OM. Do knee injuries have long-term consequences for isometric and dynamic muscle strength. Eur J Appl Physiol. 2001; 85(3-4):310-16.

37. Fitzgerald GK, Piva SR, Irrgang JJ, Bouzubar F, Starz TW. Quadriceps activation failure as a moderator of the relationship between quadriceps strength and physical function in individuals with knee osteoarthritis. Arthritis Rheum. 2004;51(1):40-8. 